\section{On the Law of Radiation.}

THE view of physicists on the question of distribution of black-body radiation is that the equipartition principle cannot apply to the problem, and that " each step from matter to ether, or back again, demanded the quantum factor, and does demand it wherever such interaction occurs," as Sir Oliver Lodge recently expressed it in NATURE, June 26, p. 89I.

In the session of February this year I presented to the Pontifical Academy of Sciences, Rome, what I think is a new method of attacking the problem, on the basis of equipartition, with only the hypothesis that frequencies are distributed in the same way as velocities in Maxwell's law for gases.

If we express the probability that frequency is between the limits $\nu$ and $\nu+d \nu$ by

$$
\theta(\nu) d \nu=C e^{-\alpha^{2} \nu^{2} \nu^{2} d \nu,}
$$

where $C=\frac{4 a^{3}}{\sqrt{ } \pi}$, the corresponding number of oscillators will be

$$
d n_{\nu}=N \theta(\nu) d \nu=\frac{4 a^{3} N}{\sqrt{\pi}} e^{-a^{2} \nu^{2}} \nu^{2} d \nu
$$

$N$ being the total number in volume unit, and the density of energy in the emitting body, on the basis of equipartition

$$
u \nu d v=\chi T N \theta(\nu) d \nu=\frac{4 a^{3} \chi T N}{\sqrt{\pi}} e^{-a^{2} \nu^{2}{ }_{\nu}^{2} d \nu .}
$$

Thence follows the expression for the emitted energy $k_{\nu} d \nu$; and assuming the Stefan law for totality of energy, it is easy to deduce $N=\frac{8 \pi \sigma T^{4}}{\chi T c}, \sigma$ being Stefan's constant, and therefore for $k_{\nu}$ the expression

$$
k_{\nu} d \nu=\frac{4 a^{3} \sigma T^{4}}{\sqrt{\pi}} e^{-a^{2} \nu^{2} \nu^{2} d}
$$

In order to calculate the constant $a$ we transfer the expression for $k_{V}$ in the corresponding $E_{\lambda}$ through the relation $E \lambda d \lambda=k_{\nu} d \nu$ and introduce Wien's law of displacement. The expression for $E_{\lambda}$ becomes

$$
E_{\lambda} d \lambda=\frac{4 a^{3} v}{\lambda^{5} \sqrt{\pi}} \lambda T e^{-\frac{a^{2}}{\lambda^{2} T^{2}}} d \lambda,
$$

where $a$ is Wien's constant multiplied by $\sqrt{2}$.

The law of distribution hence will be

$$
F(\lambda, T)=\frac{4 a^{3} \sigma}{\lambda^{5} \sqrt{ } \pi} \lambda T e^{-\frac{a^{2}}{\lambda^{2} T^{2}},}
$$

corresponding to Wien's condition, and containing only the Wien's and Stefan's constants. When $\lambda T$ is large the formula reduces to Lord Rayleigh's expression.

The new formula agrees very satisfactorily with the measured energy distribution in the solar spectrum.

Università Gregoriana, G. Gianfranceschi. Rome.

\section{The Electrical Polarity of Molecules.}

ON attempting to correlate the electrical doublerefraction (Kerr effect) of gases and vapours which has been measured by Leiser, Hansen and Szivessy, with the optical anisotropy of the molecules determined from observations on light-scattering, it is found that electrically polar molecules generally NO. 2965 , vOL. II 87 exhibit a Kerr effect which is very large in relation to their optical anisotropy. This indicates that the orientative action of the field on the molecule in such cases is chiefly due to the permanent electric doublet present in it, and is much larger than would be the case if the molecules were non-polar. In the case of molecules having an axis of optical symmetry to which the electric doublet is parallel, or is inclined at some known angle, it is possible to calculate the permanent electric moment from the value of the Kerr constant and the constant of depolarisation of the scattered light. Conversely, if the moment is known, the inclination of the electric doublet to the optic axis can be found. For example, in the case of the simple dipole molecule $\mathrm{HCl}$, we may assume the optic axis to be parallel to the doublet.

The constant of depolarisation as recently measured by Ramanathan is o.oro, and the Kerr constant from the measurements of Hansen $=0.90 \times 10^{-10}$. From this, considering the orientative action of the field to be due only to the permanent doublet, we find its moment to be $1.06 \times 10^{-18}$ electrostatic units, while if the orientative couple on the induced doublet is also taken into account as in the case of non-polar molecules, the value of the permanent moment comes out to be $\mathrm{I} \cdot 04 \times \mathrm{IO}^{-18}$. The recent determination by Zahn from dielectric constant measurements gives $\mathrm{I} \cdot 03 \times 1 \mathrm{O}^{-18}$, thus showing good agreement.

When the optical ellipsoid of the molecule has three unequal axes, measurements of the factor of depolarisation and of the Kerr constant are by themselves insufficient for an accurate determination of the electric moment. But if the moment is known from measurements of the dielectric constant, the data mentioned are of much assistance in fixing the position of the axis of the doublet. For example, if the Kerr constant of a substance is negative, we can assert definitely that the axis of the permanent doublet does not coincide with the longest axis of the optical ellipsoid. It is interesting to note in this connexion that, so far as is known, all substances having a negative Kerr constant are polar.

C. V. RAMAN.

K. S. KRISHNAN.

2ro Bowbazaar Street, Calcutta, India.

\section{Depth of Origin of the Earthquake of August 15.}

THE earthquake of Sunday morning, August I5, was one of those for which no method of determining the depth of origin had been devised until recently, and offers an opportunity for applying that which was published in the Quarterly Journal of the Geological Society for February last. The extreme limits of the area over which the shock was felt appear to have been Llandrindod Wells and Blackheath, which are about I 50 miles apart; the maximum violence seems to have just about reached the lower limit of $\mathrm{VI}^{\circ}$ of the Mercalli scale, or an acceleration of about I $50 \mathrm{~mm} . / \mathrm{sec}^{2}$ Applying the coefficients in the paper referred to, the resulting depth of origin is not far from thirty miles. This estimate is based on the newspaper reports and subject to correction when more precise data are available. The maximum acceleration may have been more than the figure adopted, the limiting value of acceleration, of a shock which is just sensible in England, may be less than the $20 \mathrm{~mm}$./ $\mathrm{sec}^{2}$ adopted, and either of these would lessen the resulting depth of origin. On the other hand, the description of the shock at 